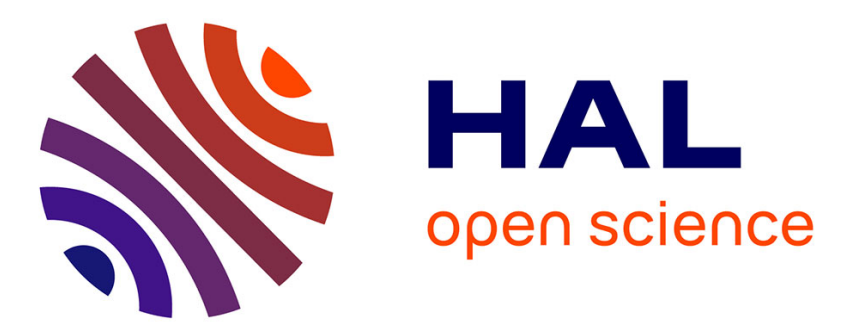

\title{
Strategy of Approach for Seizure of An Assistive Mobile Manipulator
}

Khiar Nait-Chabane, Sébastien Delarue, Philippe Hoppenot, Etienne Colle

\section{To cite this version:}

Khiar Nait-Chabane, Sébastien Delarue, Philippe Hoppenot, Etienne Colle. Strategy of Approach for Seizure of An Assistive Mobile Manipulator. Robotics and Autonomous Systems, 2009, 57, pp.222235. 10.1016/j.robot.2008.04.004 . hal-00341318

\section{HAL Id: hal-00341318 https://hal.science/hal-00341318}

Submitted on 16 Jul 2009

HAL is a multi-disciplinary open access archive for the deposit and dissemination of scientific research documents, whether they are published or not. The documents may come from teaching and research institutions in France or abroad, or from public or private research centers.
L'archive ouverte pluridisciplinaire HAL, est destinée au dépôt et à la diffusion de documents scientifiques de niveau recherche, publiés ou non, émanant des établissements d'enseignement et de recherche français ou étrangers, des laboratoires publics ou privés. 


\title{
Strategy of Approach for Seizure of an Assistive Mobile Manipulator
}

\author{
K. Nait-Chabane, S. Delarue, P. Hoppenot and E. Colle \\ IBISC-CNRS, University of Evry, France \\ Khiar.NaitChabane | Sébastien.Delarue | philippe.hoppenot | etienne.colle@ibisc.univ-evry.fr \\ Corresponding author Philippe.hoppenot@ibisc.univ-evry.fr
}

\begin{abstract}
In assistive robotics, a manipulator arm constitutes one possible solution for restoring some manipulation functions to victims of upper limb disabilities. The aim of this paper is to present a global strategy of approach of an assistive mobile manipulator (manipulator arm mounted on a mobile base). A manipulability criterion is defined to deal with the redundancy of the system. The aim is to keep the arm manipulable ie capable to move by itself. The strategy is based on human-like behaviour to help the disabled operator to understand the action of the robot. When the robot is far from its objective, only the mobile base moves avoiding obstacles if necessary. When the objective is close to the robot, both mobile base and arm move and redundancy can be used to maximise a manipulability criterion. All the situations are tested separately and a global mission is realised in which all the previous situations are encountered. The partial results obtained with the real robot consolidate the results of simulation. This paper does not propose an autonomous path planning and navigation of the mobile arm but an assistance to the user for remote controlling it.
\end{abstract}

\section{Introduction}

In assistive robotics, a manipulator arm constitutes one possible solution for restoring some manipulation functions to victims of upper limb disabilities. The literature proposes three distinct manipulator arm configurations. The first one consists of a workstation in which a manipulator arm evolves within a structured environment (RAID, AFMASTER (Busnel, 2001)). In the second configuration, a manipulator arm is added to an electrical wheelchair ((Kwee, 1993), (Evers, 2001)). The third configuration aims at expanding the field of application of manipulator arm by making it mobile, MoVAR (Van der Loos, 1995), MOVAID (Dario, 1999) and ARPH (Hoppenot, 2002). Our research deals with object manipulation by a disabled person using a mobile manipulator arm. The principle is that the machine is semiautonomous. The functional substitution is reached by a close human-machine cooperation. Each entity, person and machine, brings a contribution while trying however to reduce the person's workload and to preserve the control of the task by the user. Semi- autonomy is a compromise which is a way to design an affordable system able to be controlled by a disabled person in a partially unknown environment.

The paper describes a strategy of approach for object grasping. Different kinds of work have been done in two close fields: mobile manipulators ((Khatib, 1986) or (Padois, 2007)) and static manipulators (Bowling, 2005). In this last case, the paper deals with non-redundant manipulators with the objective of piloting accelerations, velocities and forces. In the case of mobile manipulator, meaning manipulator mounted on a mobile platform, the system is redundant which lead to particular issues debated in section 3.

The idea which will be developed in paragraph 3 is that the user provides the high level function of planning. The role of the user is to pilot the robot by the gripper i.e. to define the path by remote controlling the gripper of the robot. The remainder of the kinematic structure of the mobile robot is configured autonomously to carry out the request of the operator. Among the configurations which the robot can take, we fix certain constraints which depend on the phase of approach: these constraints can be the obstacle avoidance or choice of a configuration of the arm which approaches an anthropomorphic configuration. This last point aims at facilitating the appropriation of the system by the user (Mestre, 2005). This paper does not propose an autonomous path planning of the mobile arm but an assistance to the user for remote controlling it. The assistance strategy is divided into phases which according to situations take into account the robot redundancy. This paper starts with describing the robot assistant and the different types of control modes put at the disposal of the person to control the robot. Then a scenario based on human behaviour identifies the main stages of a seizure task. Redundancy of the mobile manipulator can be exploited to answer to specific constraints of the task which we will specify for every phases. Simulation results aim at comparing manipulability measures, especially directional manipulability we propose, and on the other hand, verifying validity, advantages and limits of the seizure strategy. The partial results obtained with the real robot consolidate the simulation results. 


\section{Architecture and control modes of assistive mobile manipulator 2.1 Assistance system architecture and robot models}

The system is divided into a robotic assistant and a man-machine interface station for remote controlling the robot (Figure 1). The robot in ARPH (Assistant Robot for People with Handicap) project is composed of a Manus arm manufactured by Exact Dynamics Company mounted on a mobile platform powered by two independent drive wheels, designed in IBISC laboratory (Hoppenot, 2002). The mobile platform is equipped with a pan tilt camera and an ultrasonic ring. The screen of the control station displays control and feedback information such as control panels for the robot and the camera, and video live.

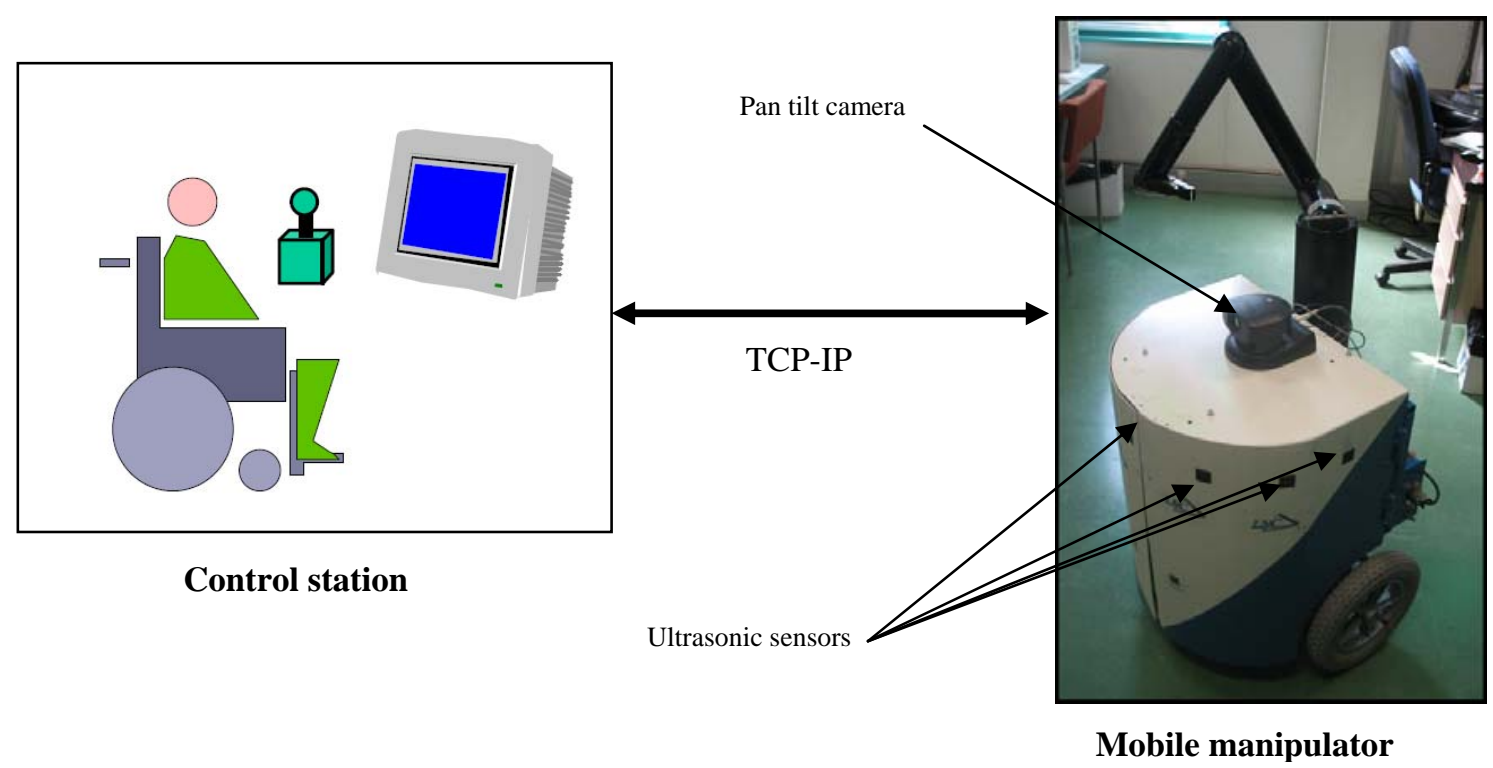

Figure 1: Assistive mobile manipulator

Manus arm can be controlled in Cartesian space with the software provided by the manufacturer in order to more easily achieve tasks. However our objective is not only the realization of an operational task, but the manner of which it must be achieved. To choose the best configuration of the manipulator arm or of the whole system by optimizing criteria, it is necessary to have the relation between operational and configuration spaces. Manus has anthropomorphic 6 dof (degrees of freedom) structure, 3 dof for the position and 3 dof for the orientation of the gripper. Manus configuration is given by the vector

$$
q_{a}=\left[q_{a 1}, q_{a 2}, \ldots q_{a b}\right]^{\mathrm{T}}
$$

Configurations space of Manus arm, noted $N_{a}$, is defined by $n_{a}=6$ generalized coordinates. The operational space, noted $M_{a}$, is described by $m_{a}=6$ operational coordinates

$$
X_{a}=\left[X_{a 1}, X_{a 2}, \ldots X_{a 6}\right]^{\mathrm{T}}
$$

For the kinematic modelling of the manipulator arm we have applied the modified formalism of Denavit Hartenberg (Yoshikawa, 1990). We have established kinematic models for the arm and the platform, then for the whole system (Nait-chabane, 2006).

\subsection{Control modes}

The user has three types of control modes at his disposal to drive robot. The first one is the manual mode. It permits the user to control all the degrees of freedom of the system. However the complexity of the task generates extra workload. In the second mode called "automatic mode", the system carries out tasks autonomously. Autonomous mode reduced the implication of the person by limiting his/her action contrary to the wish generally expressed by the users. The second defect comes from the difficulty to propose a fully autonomous system which remains affordable. Both modes do not answer to the various situations that can be met, do not take into account human factors, environment variability and do not take advantage of a true manmachine cooperation. In previous works (Hoppenot, 2002) we have developed a third type of mode called "shared control mode" that palliates faults listed above. It consists in sharing the control of robot operational coordinates between human and machine. The interest is that the implication of the person can be variable between manual and automatic modes. It can also be better to take into account human and environmental 
situations. For instance, when the robot is moving a shared control mode can consist in helping the operator in avoidance of obstacles located along the way of the robot. The user gives the main direction to be followed; the robot avoids locally obstacles if needed. In another shared mode, so called camera mode, the user controls the direction the robot must follow by orientating the pan tilt camera. A tracking function can be added to the previous mode. The movement of an object is automatically tracked by the camera. Once an object is chosen by the operator, the camera follows it and the mobile platform moves along this direction. For each of last two modes one can add obstacle avoidance described above.

\section{Strategy of approach for seizure}

\subsection{Principle and context of the strategy}

We locate our work in the context of the control shared modes whose principle is presented in paragraph 2.2. In a more global task of seizure, the principle which was adopted is that the operational coordinates of the end effector (position and orientation) are controlled by the user. The difficulty lies in the calculation of the generalized coordinates of the mobile manipulator which is a redundant system. One can completely adopt a strategy inspired of human behaviour. If one includes the research of the object to seize, a task of seizure can be divided into three stages in which constraints are different. The first stage consists in moving inside the residence to find an object which is in a place not necessary known by the person. In this stage, the obstacle avoidance is enabled. When the object is in sight, the stage of approach begins. Anticipation is brought into play to start to position the body and the arm according to characteristics of the object (form, position, close environment, in particular cases of obstruction or potential obstacles (Jeannerod, 1984), (Land, 1994), (Grasso,1998)). In these first two stages, the mobility is privileged, the arm remaining on standby in such a way it does not interfere with the robot navigation. Last stage is a precise approach which makes it possible to refine the situation of the hand compared to the points of object seizure when this one is close. Seizure itself and following stages are not treated in this paper.

A similar strategy is possible if it is considered that the mobile robotized arm is an extension of the human arm. By analogy the mobile platform of the robot corresponds to the body of the person, Manus arm to human arm and end effector of Manus to user's hand. This way of tackling this problem of strategy falls under the approach which was developed in our laboratory in previous works relating to the appropriation (Rybarczyck, 2002). One of the ideas defended in this approach is that it is necessary, as far as possible, that the person in situation of teleoperation is able to interpret what robot is doing. One of the means of reaching that goal is to develop a "bio inspired" robot, at the structural, behavioural and functional levels. It is not the object of this paper to defend this point of view. We will retain only that we adopt a bio-inspired strategy which can be broken up into two stages: - a stage of research and approach of the object in which only the mobile base is involved. Obstacle avoidance is activated when it is necessary. In that stage, the arm keep a fixed position, chosen with two aims in mind: (i) keep the arm away from its singular positions to prepare the final seizure in which it is important to have at disposal the maximum degrees of freedom of the arm for fine movements and (ii) put the arm in a position which does not disturb the mobile platform navigation.

- a stage of fine approach in which the mobile platform and the manipulator arm are involved. In that stage, the redundancy of the system ( 6 degrees of freedom in space for 8 degrees of command -6 for the arm and 2 for the mobile platform) can be used to satisfy constraints.: obstacle avoidance and manipulability optimisation. This last constraint permits to keep at disposal the maximum degrees of freedom of the arm for fine movements to prepare the final seizure.

"In the human robot cooperation that we propose the autonomous functions of the robot can be regarded as a specific assistance of the user according to the situation defined by the phase of approach in which the robot is running. There are two principal phases: far objective and near objective. The swap from the first phase to following one is specified by the user. These phases themselves are divided into two zones: robot close to an obstacle or robot distant from an obstacle. The swap between the zones is automatic. It depends on the distance between the robot and the obstacle measured by the ultrasonic ring. When the objective is far from the robot, only the platform is active, the arm remains in a configuration which does not obstruct the manoeuvrability of the robot. The exploitation of the redundancy is unnecessary in this case. When the objective is close to the robot, all the degrees of freedom of the mobile arm are involved.

The interest of this approach is to leave to the user the control of the robot while facilitating the task to him.” The objective of the paper is to calculate the corresponding generalized coordinates by adopting a resolution method according to the stage of scenario in which the robot is. As soon as the intervention of the manipulator arm appears necessary, it is possible to exploit the redundancy of the whole system for both satisfying operational task and some additional constraints. Terminology adopted in the paper is summarized below:

- Operational task (OT): put the end-effector (EE) of the mobile manipulator in situation of grasping the object.

The strategy presented in the paper is only interested in the approach for the seizure. 
- Primary task: to be executed firstly. It can be an operational task or another task according to the strategy.

- Secondary task: to be executed by using redundancy (if possible).

- Additional task: to be executed with the same importance as the Primary task.

- Obstacles avoidance constraint. It only concerns the avoidance of obstacles by the mobile platform.

- Manipulability constraint. Redundancy is exploited to optimize manipulability measure.

\subsection{Phases}

An analysis of the scenario described previously brought to differentiate in stages two notions: phase and zone. The scenario is decomposed then into two phases according to whether the objective, namely the object to be seized, is distant or close. In each phase, two zones are considered, near or distant from an obstacle, because the presence of the obstacle modifies the behaviour of the robot. The phase change is specified by the operator while the zone change is determined automatically thanks to obstacle detection.

\subsubsection{Phase: far objective}

As it was specified previously, when the object to be reached is far, the task is summarized to navigation of the mobile platform under the constraint of obstacle avoidance. The passage from a zone without obstacles to a zone with obstacles is defined by a threshold on the ultrasonic sensors measures (see Figure 2). The choice of this threshold is determined by several factors: characteristics of perception system, capacities of the mobile manipulator and control mode selected. This is not an easy problem. We suppose the value of this threshold is known. It is called T1.

Threshold: T1

\begin{tabular}{l|l} 
Zone : far obstacle & \multicolumn{1}{c}{ Zone : close obstacle } \\
\hline Primary task: operational task (OT) & $\begin{array}{l}\text { Primary task: combination of obstacles avoidance and } \\
\text { convergence towards the objective }\end{array}$
\end{tabular}

Figure 2: Far objective

In this phase the mobility function is privileged, the manipulator arm remaining at a rest position which doesn't interfere with the manoeuvrability of the robot during navigation. It is useless to manipulate joints of the manipulator arm since no operation of seizure is in progress. Gripper control only involves a movement of the mobile platform in direction of the goal while passing by the intermediate points in order to approach the seizure zone. Degrees of freedom of the platform are used to accomplish the operational task imposed to gripper i.e. "go in the direction of the goal". No redundancy is however available to take into account additional constraints. As soon as the threshold "T1" is crossed, it is necessary to establish an area of safety which avoids the collision with the environment. In this case obstacle avoidance becomes the primary task according to the distance to obstacles.

\subsubsection{Phase: close objective}

In this case the user informs the system that the object to be seized is close. As it has been described in the scenario, all of the degrees of freedom of the mobile manipulator (6 dof of the arm and 2 dof of the mobile platform) are put at contribution. In this phase, one also distinguishes two zones according to the distance from the obstacles. In the zone "far obstacle", before threshold T2 (Figure 3), if the environment is not too much cluttered, the mechanism of anticipation identified in the scenario can be used to direct the mobile manipulator in order to facilitate the future seizure. The operational task remains the displacement of the gripper according to intermediate points imposed by the control mode. However the redundancy makes it possible to satisfy additional constraints, in particular a better positioning of the mobile manipulator for the next seizure.

Redundancy of the system is exploited to optimize the manipulability of the manipulator arm or the mobile manipulator and to answer the constraint of obstacle avoidance. As soon as threshold 2 is crossed, by safety measure, linear displacements of the platform are prohibited. The operational task consists in positioning the gripper in a situation of seizure of the object with the best value of a manipulability criterion, presented below. The crossing of threshold $\mathrm{T} 2$ is detected by ultrasonic ring. 


\section{Zone: far obstacle}

Primary task: OT

Secondary tasks: manipulability and obstacles avoidance
Threshold: T2

\section{Zone: close obstacle}

Primary task: OT

Secondary task: manipulability

Additional task: platform linear velocity $v$ equal to zero

Figure 3: Close objective

\section{Manipulability measures}

\subsection{State of the art}

A well-established tool used for the motion analysis of manipulators is known as the manipulability ellipsoid approach. The concept of manipulability was originally introduced by Yoshikawa ((Yoshikawa, 1985), (Yoshikawa, 1990)) for manipulator arm, in order to denote a measure for the ability of a manipulator to move in certain directions. The set of all end-effector velocities that may be attained by joint velocities such that the Euclidean norm of $\dot{q}_{a}\left(\left\|\dot{q}_{a}\right\|=\left(\dot{q}_{a 1}^{2}+\dot{q}_{a 2}^{2}+\cdots \dot{q}_{a n}^{2}\right)^{1 / 2}\right)$ satisfying $\left\|\dot{q}_{a}\right\| \leq 1$ is an ellipsoid in $m$-dimensional Euclidean space. This ellipsoid represents the manipulation ability and is called the "manipulability ellipsoid".

Yoshikawa defines the manipulability measure $w$ as follows:

$$
w=\sqrt{\operatorname{det}\left(J_{a}\left(q_{a}\right) J_{a}^{T}\left(q_{a}\right)\right)}
$$

which can be simplified into $w=\left|\operatorname{det}\left(J_{a}\left(q_{a}\right)\right)\right|$ when $J_{a}\left(q_{a}\right)$ is a square matrix.

Let's now consider the singular value decomposition of $J_{a}$, as given by:

$$
J_{a}=U_{a} \sum_{a} V_{a}^{T}
$$

where $U_{a} \in R^{m \times m}$ and $V_{a} \in R^{n \times n}$ are orthogonal matrices, and:

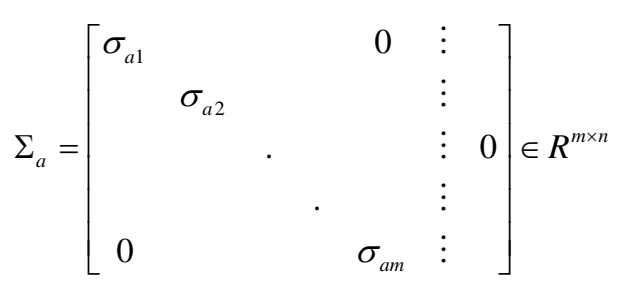

in which: $\sigma_{a 1} \geq \sigma_{a 2} \geq \cdots \geq \sigma_{a m}$.

The value of $w=\sigma_{a 1} \cdot \sigma_{a 2} \cdots \sigma_{a m}$ is proportional to the ellipsoid volume.

Another measure has been proposed for characterizing the distance of a configuration from a singularity (Salisbury, 1982). This measure is given by:

$$
w_{2}=\frac{\sigma_{a m}}{\sigma_{a 1}}
$$

where $\sigma_{a_{1}}$ and $\sigma_{a_{m}}$ are the maximum and minimum singular values of the Jacobian matrix, respectively.

Bayle (Bayle, 2001) defined a measure $w_{5}$ that extended the notion of eccentricity of the ellipse, i.e.:

$$
w_{5}=\sqrt{1-\frac{\sigma_{a m}^{2}}{\sigma_{a 1}^{2}}} .
$$

\subsection{New manipulability: directional measure}


All of the above mentioned measures describe system manipulability without taking the task of the manipulator into account. They do not include information about either the task or the direction the end-effector required to move. A new measure should therefore be introduced to include such information.

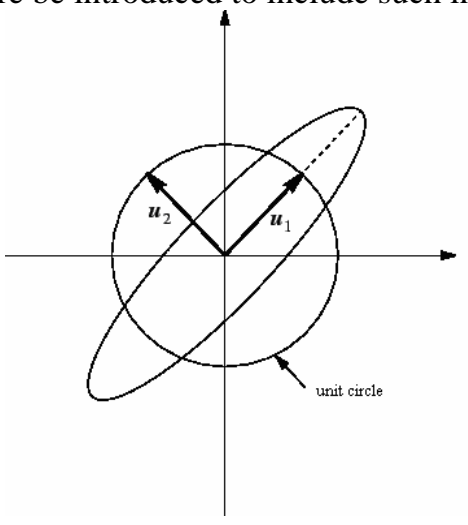

Figure 4: Manipulability ellipse in the two-dimensional case.

The Singular Value Decomposition (4) of the Jacobian matrix and its geometric relationship offer further insights into characterizing the manipulability of mechanical systems. Let $u_{a i}$ be the $i^{\text {th }}$ column vector of $U_{\mathrm{a}}$. The primary axes of the manipulability ellipsoid are then: $\sigma_{a 1} u_{a 1}, \sigma_{a 2} u_{a 2}, \cdots \sigma_{a m} u_{a m}$. Figure 4 provides an illustration of the two-dimensional case, according to which $u_{1}$ and $u_{2}$ yield the major and minor ellipse axes, respectively. We propose to include information on the direction of the task wished to precisely know the manipulation capacity of the manipulator arm for the execution of this operational task.

Let $\dot{X}_{d}$ be the desired task. We now define a unit vector $d=\frac{\dot{X}_{d}}{\left\|\dot{X}_{d}\right\|}$, which gives the direction of the imposed task.

By using properties of the scalar product and the singular values that represent radius of the ellipsoid, we define a new manipulability measure as being the sum of the absolute values of the scalar products of the directional vector of the task by the singular vectors pondered by their corresponding singular values. This new measure is noted $w_{d}$ (Nait-Chabane, 2007).

$$
w_{d}=\sum_{i=1}^{m}\left|\left(d^{T} \cdot u_{a i}\right) \sigma_{a i}\right|
$$

The measure is maximized when the manipulation capacity of the arm along the direction of the task imposed is maximal. It is equal to zero if there is no possibility of move along this direction.

\section{Methods of resolution according to phase in progress}

From a given generalized configuration $q$, the objective is to calculate the differential $d q$ of the generalized coordinates corresponding to a differential $d X$ of operational coordinates provided by the control mode. Among kinematic inversion methods, numerical methods are more general notably those based on the pseudo inverse. The interest of this paragraph lies in the adaptation of this method to the more general problem of the strategy of seizure that we propose. Like seen previously, according to the phase in progress, there is possibility to take advantage of the system redundancy to satisfy certain constraints which it is possible to optimize. We will specify for each phase the operational coordinates which one wants to control and the resolution method.

\subsection{Phase : far objective}

\subsubsection{Zone : far obstacle}

Primary task: operational task

Secondary tasks: nothing

Additional task: nothing

One recalls that in this phase no redundancy is available. The manipulator arm remains at rest in a position which does not interfere with mobile-platform motion. It is enough to control the platform from a point which coordinates are those of the gripper expressed in the platform reference frame. Equations giving the position of 
the gripper according to the orientation of the platform and the position of the arm manipulator on the platform are written as follows:

$$
\left\{\begin{array}{l}
x_{1}=x_{P}+\left(x_{a 2}+a\right) \cos \left(\theta_{p}\right)-\left(b-x_{a 1}\right) \sin \left(\theta_{p}\right) \\
x_{2}=y_{p}+\left(x_{a 2}+a\right) \sin \left(\theta_{p}\right)+\left(b-x_{a 1}\right) \cos \left(\theta_{p}\right) \\
x_{3}=x_{a 3}+c=\text { constant }
\end{array}\right.
$$

Where $x_{a i}(i \in\{1,2,3\})$ are end-effector coordinates expressed in the manipulator arm frame and a, b and c (constants) are arm-base coordinates expressed in the platform frame. $x_{p}, y_{p}, \theta_{p}$ are platform operational coordinates expressed in the world frame and $\mathrm{x} 1, \mathrm{x} 2, \mathrm{x} 3$ are the coordinates of the gripper in the global frame. These three coordinates are sufficient to define the operational task of the gripper since the mobile platform can move only in the horizontal plane. The model giving the position of the gripper in the global frame is only useful for autonomous functions. Indeed in the shared control mode, the user directly teleoperates the position and the orientation of the gripper. The management of the redundancy only acts on degrees of freedom of the mobile platform and the first three generalized coordinates of the manipulator arm which define the gripper position. The inversion scheme of the system is simple in this case.

$$
\dot{X}_{d}=\bar{J}(q) u
$$

where :

$$
\begin{aligned}
& \dot{X}_{d}=\left[\dot{x}_{1 d}, \dot{x}_{2 d}\right]^{T} \text { et } u=[v, \omega]^{T} \text { (platform lineaire and angular velocities) } \\
& \bar{J}(q)=\left[\begin{array}{ll}
c_{\theta_{p}} & -\left(x_{a 2}+a\right) s_{\theta_{p}}-\left(b-x_{a 1}\right) c_{\theta_{p}} \\
s_{\theta_{p}} & \left(x_{a 2}+a\right) c_{\theta_{p}}-\left(b-x_{a 1}\right) s_{\theta_{p}}
\end{array}\right]
\end{aligned}
$$

with $x_{a i}(i \in\{1,2,3\}$ ) end-effector coordinates expressed in the reference frame of the manipulator arm and a, b and c arm-base coordinated expressed in the platform frame. $c_{\theta_{p}}, s_{\theta_{p}}$ are cosine and sine of $\theta_{p}$ angle, respectively. So that a solution exists, it is necessary that the rank be equal to 2 and thus that $x_{a 2+} a$ be different from zero. Gripper must not be therefore aligned along wheels axis.

\subsubsection{Zone : close obstacle}

Primary task: Local navigation

Secondary task: nothing

Additional task: nothing

Local navigation is based on the combination of two reactive behaviours, obstacles avoidance and convergence of the platform towards the goal. The importance of every one of the two behaviours is variable according to the distance to obstacles. Main principles of the approach developed at laboratory are shortly described below. For more details see the following references ((Benreguieg, 1997), (Hoppenot, 1996)).

\subsubsection{Obstacle avoidance}

The platform of ARPH project is equipped of a ring of ultrasonic sensors which makes it possible the detection of obstacles in cluttered environments. The method consists in valuing the cluttered space all around the robot. One tries then to make evolve the robot in the less cluttered direction.

The navigation algorithm is based on a fuzzy controller (Figure 5). Inputs are respectively $R n, L n$ and $F n$, the normalized distances between the robot and the nearest environment along right, left and frontal directions:

$$
R_{n}=\frac{R}{R+L}, L_{n}=\frac{L}{R+L} \text { and } f_{n}=\frac{f}{\sigma}
$$

$L, R$ and $F$ are respectively Left, Right and Front measures and, $\sigma$ is the distance of influence of the sensors. 


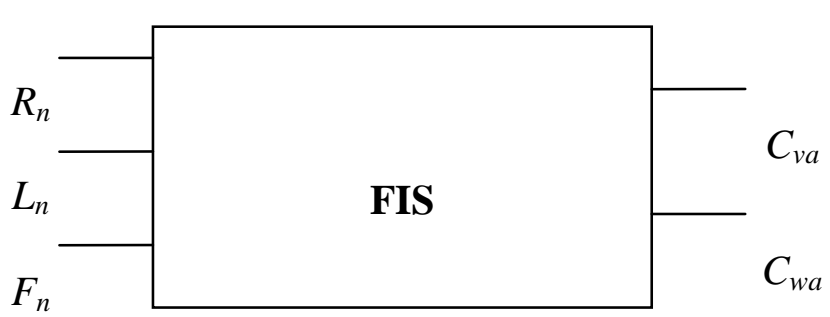

(a)

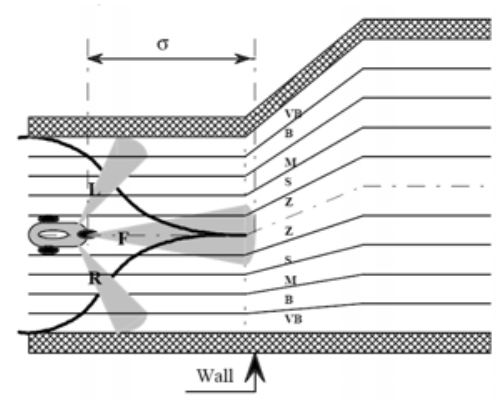

(b)

Figure 5 : Navigation based fuzzy controller: a) FIS, b) evolution of the partition universe of discourse for distance measures

Outputs variables $C_{v a}$ et $C_{\omega a}$ are the multiplying coefficients of linear and angular velocities of the platform. The universe of discourse is $-1 \leq C_{\omega a} \leq 1$ and between 0 and 1 for other cases. Membership functions are triangular. Linguistic repartitions of variables are:

$-C_{v a}=\{\mathrm{Z}$ : zero ; S : Small ; M : Medium; B : Big ; VB: Very Big $\}$

- $C_{o d}=\{$ NB : negative Big ; NS : negative Small ; Z : Zero ; PS : Positive Small ; PB : Positive Big $\}$

\subsubsection{Convergence toward the goal}

We apply the concept of potential field method (Khatib, 1986). To reach a goal the robot is attracted by a force which guides it to destination. Actions (Cvg, C $\omega$ g) generated by this force are modulated by the distance $\left\|P, S_{\text {goal }}^{i}\right\|$ between the centre P of the robot and the sub-goal (Erreur ! Source du renvoi introuvable.). $\theta_{\mathrm{g}}$ is the deviation needed to reach $S^{k}$. D is the distance of influence of the goal $S_{\text {goal }}^{i}$.

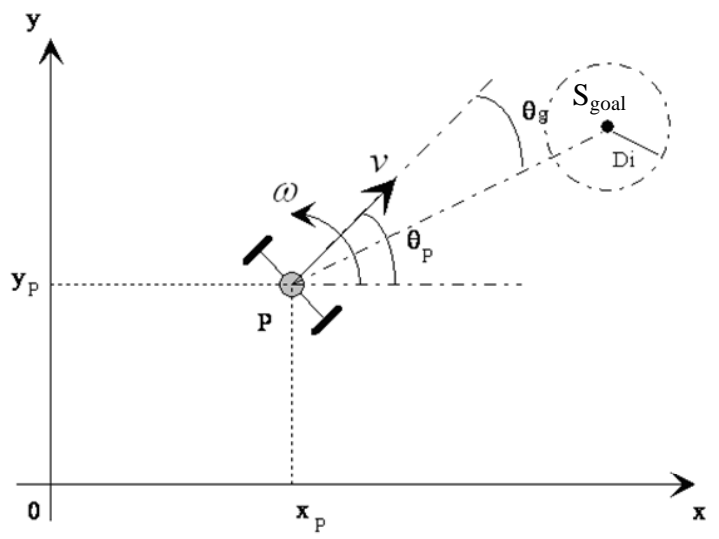

Figure 6: convergence towards goal.

When the robot is far enough from the sub-goal the coefficient of the angular velocity is:

$C_{\omega g}=\frac{C_{g}}{\left\|P, S_{\text {goal }}^{i}\right\|} \times \frac{D}{\pi} \cdot \theta_{g}$.

$C g$ coefficient (between -1 and 1) is chosen so that the robot reaches a maximal angular speed for $\theta_{g}<\pi$ so that its direction does not deviate too much from the direction of the straight line $P S_{\text {goal }}^{i}$.

But, if the robot is in the influence zone of a sub-goal, the coefficient of the angular velocity becomes $C_{\omega g}=\frac{C_{g}}{\pi} \theta_{g}$ for avoiding too important angular velocity. 
Moreover the coefficient of regulating of linear velocity depends on angular velocity coefficient by the following relation, $C_{\mathrm{vg}}=1-\left|C_{\omega g}\right|$. It expresses the following rule, the more the robot is oriented toward the direction of the goal, the more it can go quickly.

\subsubsection{Behaviour fusion}

Obstacles avoidance and convergence toward the goal are merged to ensure both robot safety and convergence toward the goal:

- If a very close obstacle is detected on the sides or in front of the robot, obstacle avoidance has priority and attraction towards the goal is cancelled, $C_{\omega g}=0$.

- In the contrary, the angular speed $\omega_{\mathrm{r}}$ applied to the robot results from a linear combination of coefficients provided by "search for free space" and "attraction by the goal" modules:

$$
\omega_{e}=\left(\alpha \times C_{\omega a}+\beta \times C_{\omega g}\right) \times \omega \max .
$$

where $\alpha$ et $\beta$ are adaptable coefficients chosen by experimentation to have the best trajectory.

In the same way, the linear velocity $\mathrm{V}_{\mathrm{e}}$ is given by

$\mathrm{v}_{\mathrm{e}}=\min \left(\mathrm{C}_{\mathrm{va}}, \mathrm{C}_{\mathrm{vg}}\right) \times \mathrm{V}_{\max }$ if the distance $\mathrm{d}\left(P, S_{\text {Goal }}^{i}\right)>\mathrm{D}$

or $\mathrm{v}_{\mathrm{e}}=\min \left(\mathrm{C}_{\mathrm{va}}, \mathrm{Cvg}_{\mathrm{vg}}\right) \times \mathrm{V}_{\min }$ if the distance $\mathrm{d}\left(P, S_{G o a l}^{i}\right)<\mathrm{D}$,

where $\mathrm{V}_{\max }$ et $\mathrm{V}_{\min }$ are respectively the maximum and the minimum linear velocities.

\subsection{Phase: close objective}

\subsubsection{Zone: far obstacle}

Primary task: Operational task

Secondary tasks: linear combination between obstacle avoidance task and manipulability optimisation Additional task: nothing

In this phase the manipulator arm and the mobile platform are implied in the execution of the operational task. The calculation of joint velocities is based on the extension of kinematics inversion of manipulators arm to the case of the mobile manipulators. The non-holonomic constraint of the platform is taken into account by introducing the notion of reduced model of transformation ((Foulon, 1998), (Foulon, 1999), (Bayle, 2001a)). So, this formulation permits to include all kinematic constraints in a same model.

General solution permitting to calculate control velocities is given by:

$$
u=\bar{J}^{+} \dot{X}_{d}+\left(I_{n-1}-\bar{J}^{+} \bar{J}\right) Z
$$

where $u=\left(v, \omega, \dot{q}_{a 1}, \dot{q}_{a 2}, \dot{q}_{a 3}, \dot{q}_{a 4}, \dot{q}_{a 5}, \dot{q}_{a 6}\right)^{T}$ represents the first two components are respectively linear and angular velocities of the platform and the six last components are joint variables of the arm, $\dot{\boldsymbol{X}}_{\boldsymbol{d}}$ defines operational task, $\bar{J}$ is the Jacobian matrix and $Z$ is an arbitrary vector.

A first possibility is to use the vector $\mathrm{Z}$ to optimize a function depending on the configuration of the system (gradient method) (Bayle, 2001b), (Bayle, 2001a).

$$
Z=-K\left(\frac{\partial P(q)}{\partial q} M\right)^{T}
$$


where $P(q)$ is the function to be minimized, $\mathrm{K}$ is a positive constant and $M\left(\theta_{P}\right)=\left[\begin{array}{ccc}\cos \left(\theta_{P}\right) & 0 & \\ \sin \left(\theta_{P}\right) & 0 & 0_{3 \times n_{B}} \\ 0 & 1 & \\ 0_{n_{B} \times 2} & & I_{n_{B} \times n_{B}}\end{array}\right]$

The second possibility we propose consists in extending the formulation proposed by (Chiaverini, 1997) for the redundant manipulator arms. When the second task $T_{s}$ is defined by $T_{s}=\left(\bar{J}_{s}(q), \dot{X}_{s, d}\right)$, equation (12) is written in the following form:

$$
u=\bar{J}^{+} \dot{X}_{d}+\left(I_{n-1}-\bar{J}^{+} \bar{J}\right) \bar{J}_{s}^{+} \dot{X}_{s, d}
$$

One can combine the realization of several secondary tasks while using the same principle. In the case of real system it is more interesting to incorporate the feedback data provided by sensors in the algorithm. A closed loop version of the previous equations can be adopted. Equation (10) becomes:

$$
u=\bar{J}^{+}\left(\dot{X}_{d}+k_{p} e_{p}\right)+\left(I_{n-1}-\bar{J}^{+} \bar{J}\right) \bar{J}_{s}^{+}\left(\dot{X}_{s, d}+k_{s} e_{s}\right)
$$

In our case two secondary tasks can be considered, obstacles avoidance and manipulability to be optimized. The idea is to calculate the linear combination of manipulability function with obstacle avoidance:

$$
u=\bar{J}^{+} \dot{X}_{d}+\left(I_{n-1}-\bar{J}^{+} \bar{J}\right)\left(\alpha\left(K \frac{\partial P(q)}{\partial q} M\right)^{T}+(1-\alpha) \bar{J}_{s}^{+} \dot{X}_{s, d}\right)
$$

$\alpha \in[0,1]$ is a weighting coefficient. When $\alpha$ is equal to 1 , secondary task concerns only the manipulability optimization and when $\alpha$ is equal to 0 , secondary task concerns only the obstacle avoidance.

$\mathrm{P}(\mathrm{q})$ is the manipulability function to be maximized which is one of manipulability measures presented above. Obstacle avoidance only uses mobile platform coordinates. If one respects the formulation of (Chiaverini, 1997), the avoidance obstacle secondary task is described by the task vector (operational velocities) and the Jacobian matrix as follows:

$$
\dot{X}_{s, d}=\left[\begin{array}{lll}
v_{e}, \omega_{e} & 0_{1 \times n_{a}}
\end{array}\right]^{T}, \bar{J}_{s}=\left[\begin{array}{cc}
I_{2 \times 2} & 0_{2 \times n_{a}} \\
0_{n_{a} \times 2} & 0_{n_{a} \times n_{a}}
\end{array}\right]
$$

If one is only interested in the position of the OT, only the first three joints of the manipulator arm are used $\left(n_{a}=3\right)$.

$\mathrm{v}_{\mathrm{e}}, \omega_{\mathrm{e}}$ are respectively linear and joint velocities of the platform provided by obstacles avoidance module described previously.

Finally,

$$
u=\bar{J}^{+} \dot{X}_{d}+\left(I_{n-1}-\bar{J}^{+} \bar{J}\right)\left(\alpha\left(K \frac{\partial P(q)}{\partial q} M\right)^{T}+(1-\alpha)\left[v_{e}, \omega_{e}, 0_{1 \times n_{a}}\right]^{T}\right)
$$

$\alpha \in[0,1]$ is a weighting coefficient, $P(q)$ a function to be maximized and $\mathrm{K}$ a positive gain.

\subsubsection{Zone: close obstacle}

Primary task: Operational task.

Secondary task: Manipulability.

Additional task: linear speed of the platform is always equal to zero.

Compared to previous case, obstacle avoidance is no more a secondary task. In order to avoid collisions with the obstacles as soon as threshold 2 introduced previously is crossed, linear displacements of the platform are prohibited by imposing null linear speed. A way of solving the problem consists in adding to the operational task an additional task of the same priority:

$$
\bar{J}_{a}=\left[\begin{array}{lll}
1 & 0 & 0_{1 \times n_{a}}
\end{array}\right]
$$


Thus, the general solution becomes:

$$
\boldsymbol{U}=\overline{\boldsymbol{J}}_{t}^{+}\left[\begin{array}{c}
\dot{\boldsymbol{X}}_{d} \\
0
\end{array}\right]+\left(\boldsymbol{I}_{n-1}-\overline{\boldsymbol{J}}_{t}^{+} \overline{\boldsymbol{J}}_{t}\right) \mathbf{Z}
$$

with $\overline{\boldsymbol{J}}_{\boldsymbol{t}}=\left[\begin{array}{c}\overline{\boldsymbol{J}} \\ \overline{\boldsymbol{J}}_{\boldsymbol{a}}\end{array}\right]$ and $Z=K\left(\frac{\partial P(q)}{\partial q} M\right)^{T}$

$P(q)$ a function to be maximized (one of manipulability measures presented above) and $M\left(\theta_{P}\right)$ matrix defined in equation (13) such as $\dot{q}=M\left(\theta_{P}\right) u$.

\section{Simulation results}

The objective of simulation is double: i) to illustrate the effectiveness of the strategy of approach for the seizure, ii) to show the interest to exploit redundancy of the robot by introducing variable constraints according to the phase in progress for solving the problem of the kinematic inversion. Simulation is performed by MATLAB ${ }^{\circledR}$ software.

The goal of the simulated mission is the displacement from a given point of residence toward the object without however carrying out the final grasping of this object. Simulation concerns only the position (except orientation) of the gripper. The task is described by a set of intermediate points expressed in the operational coordinate space. The movement between two intermediate points is a straight line. We only illustrate the case where the objective to be reached is close. Combined motion of the mobile manipulator contributes to carry out the desired task. In this case, the redundancy is exploited differently according to whether the mobile manipulator is in a zone far from or close to the object (objective).

\subsection{Experimental protocol}

The protocol remains the same in all the simulation section. Let's consider known the initial operational configuration of the mobile manipulator and the intermediate and final operational coordinates of the gripper. Moreover let's consider that the operational trajectory of the gripper is carried out in a plan at a fixed height. The operational trajectory of the gripper is generated as follows: for each iteration, the distance $\Delta$ between the gripper and the nearest sub-goal are calculated. The gripper speed along the trajectory remains constant.

$$
\begin{aligned}
& \Delta_{x}=x_{S G i}-x_{1}(i) ; \\
& \Delta_{y}=y_{S G i}-x_{2}(i) ; \\
& \Delta=\sqrt{\Delta_{x}^{2}+\Delta_{y}^{2}} ; \\
& \dot{x}_{1}=V_{o p} \frac{\Delta_{x}}{\Delta} ; \dot{x}_{2}=V_{o p} \frac{\Delta_{y}}{\Delta} \\
& \text { with }: V_{o p} \text { the deplacement velocity along the trajectoiry } \\
& \dot{X}_{d}=\left[\begin{array}{lll}
\dot{x}_{1} & \dot{x}_{2} & 0
\end{array}\right]^{T}
\end{aligned}
$$

$x_{1}(\mathrm{i})$ and $x_{2}(\mathrm{i})$ are the operational coordinates of the gripper in the plan at the $\mathrm{i}^{\text {th }}$ iteration, $x_{S G i}$ and $\mathrm{y}_{\mathrm{SGi}}$ are the operational coordinates of the sub-goal and $\dot{X}_{d}$ the operational speed imposed to the gripper.

For every examples, we represent in the same figure the trajectories of the end effector EE (straight line shape) and of the platform. In another figure, we present the evolution of manipulability measure $w$ of manipulator arm while the the task is performed. w measure s chosen as reference for characterizing the intrinsic capacities of manipulation of manipulator arm in a given configuration. The initial position of the gripper and the position of the intermediate points are represented by stars on EE trajectory. The orientation of the platform during displacement is represented by triangles. Orientation is given with respect to the $\mathrm{x}$ axis of the world frame.

\subsection{Phase: close objective}

\subsubsection{Zone: far obstacle}

Mobile manipulator is close to seizure zone but at a distance higher than threshold T2 from the obstacle. In this zone, both parts of the robot contribute to the imposed-task execution. Redundancy is exploited simultaneously for obstacle avoidance and for optimizing a manipulability measure. Several manipulability measures have been 
compared taking into account either the only manipulator arm or the whole system. In the paper, examples focus on results using the measures of the manipulator arm alone $w, w_{d}$. We present above an example among several simulations tested. In equation 18, we put $\alpha=0.5$. The initial configuration of mobile manipulator is $q_{i}=[0.4,1,130,270,135.8,240]^{T}$. The operational task consists in reaching a goal following a straight line. The goal is situated at $10 \mathrm{~cm}$ from the table border. Its position in the world frame is $X_{\text {Goal }}=[1.9 ; 1.5,0.4]^{\mathrm{T}}$.

Operational speed of displacement along the trajectory is fixed to Vop $=0.05 \mathrm{~m} / \mathrm{s}$. Figure 7 gives the simulation environment and the initial configuration of the arm.

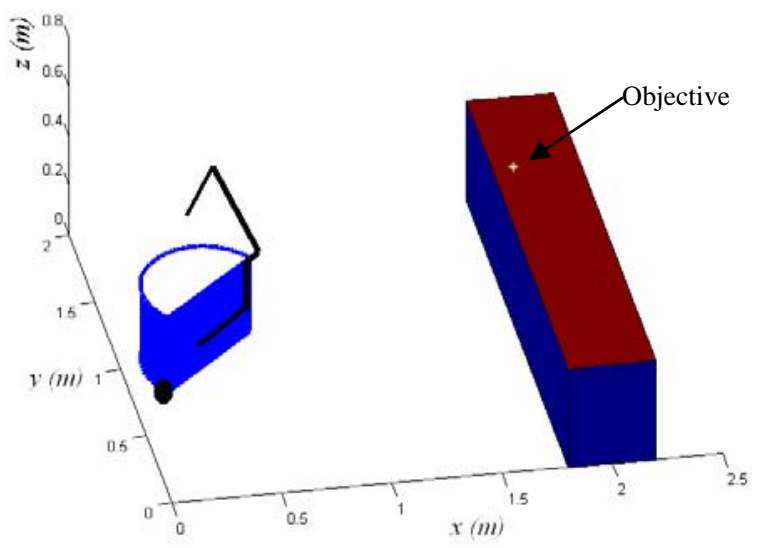

Figure 7 : Initial conditions of simulation.

\section{a. Optimization of arm manipulability $w$}

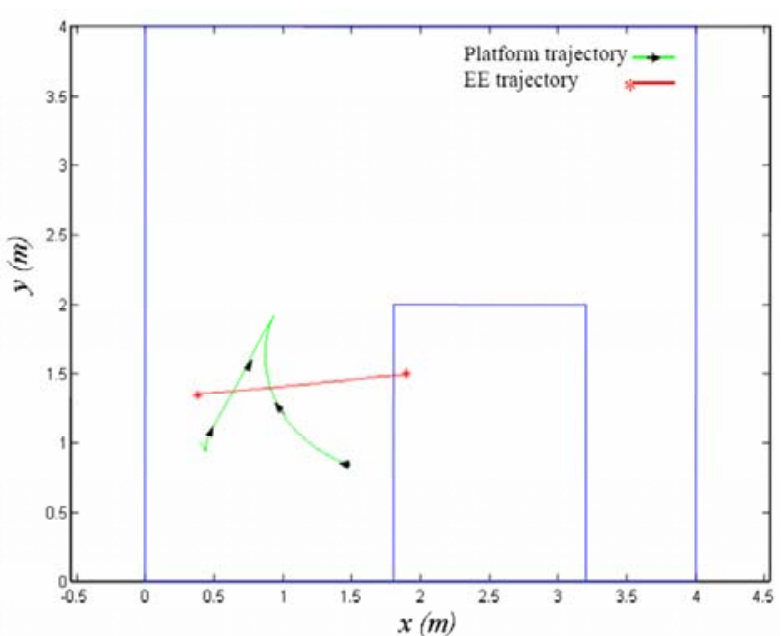

(a)

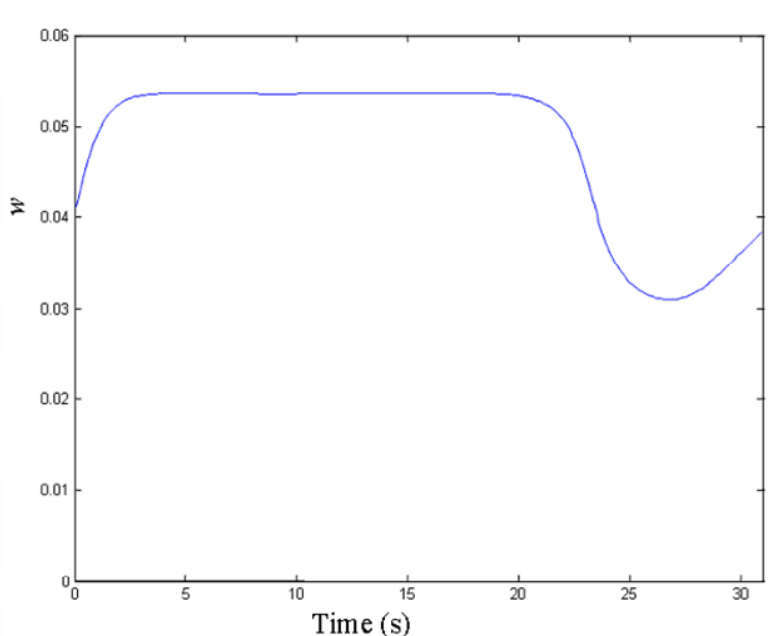

(b)

Figure 8: Optimization of arm manipulability measure $w$ and obstacle avoidance

a) Platform and EE trajectories, b) Evolution of the manipulability measure $w$.

Figure 8a shows platform and EE trajectories. The platform moves back a little at the beginning to contribute to the improvement of arm manipulability. Once the arm manipulability is improved, the platform goes forward and moves away from the obstacle. Arm manipulability (Figure 8b) is degraded slightly so the platform must reverse for continuing task while maintaining a good manipulability. EE carried out correctly the imposed task, but the final orientation of platform is opposed direction to the obstacle $\left(\theta_{p f}=163^{\circ}\right)$. Platform displacement in reverse gear is not wished: in this case, the robot carries out its movement as a blind man because the robot ultrasonic sensors are placed in the front side of the platform. 


\section{b. Arm directional manipulability optimization $w_{d}$}

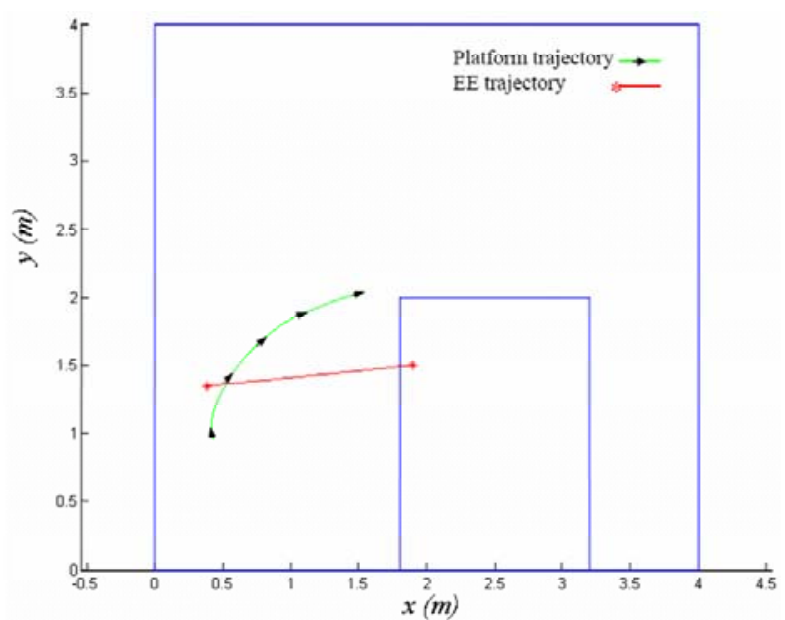

(a)

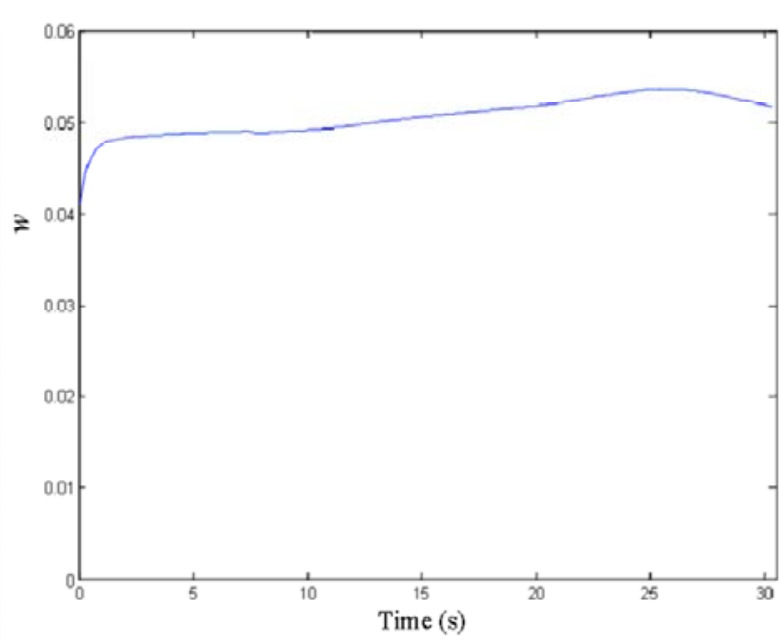

(b)

Figure 9 : Optimization of arm directional manipulability $w_{d}$ and obstacle avoidance

a) Platform and EE trajectories, b) Manipulability measure $w$.

Figure 9 illustrates the results obtained by optimizing the directional manipulability of the arm. The other secondary task is the obstacle avoidance. According to Figure 9a, one notes that platform moves back at the beginning to contribute to the improvement of arm manipulability. Contrary to the previous case illustrated in Figure $8 \mathrm{a}$ the platform continues the task moving forward according to task direction $\left(\theta_{p f}=12^{\circ}\right)$. Figure $8 \mathrm{~b}$ shows that the system carried out the task with configurations corresponding to a good arm manipulability. EE approaches the goal while following the desired trajectory, but we terminate the task execution because the detection of an obstacle at lower distance than threshold 2 from the obstacle. This problem will be solved with adding the case of close obstacle zone.

\section{Discussion}

We have tested the algorithm in various conditions. The examples which were selected to illustrate the mobile manipulator behaviour are representative of the possible situations.

One can compare measures according to three criteria:

- Task success

- Manipulation capacity of the arm at the end of the task. The question is to know under which dexterity conditions robot will begin the next task

- Platform trajectory. Forward movements of the platform with following smooth trajectory are important regarding the sensing capacities of the mobile platform. It is also a more natural behaviour, which facilitates Human-Machine Co-operation.

Manipulability measure related to the arm alone bring answers which satisfy the first two criteria by noting however a temporary manipulability reduction occurs during the task execution in $w$ case optimisation.

In term of platform behaviour, obstacle induces a reverse gear of the platform. This fact is a disadvantage considering the system moves as a blind man (there are no ultrasonic sensors in back side of the platform). Among tested measures, one notes that the arm directional manipulability $w_{d}$ makes it possible to bring satisfactory answers without graining point nor reverse gear. When the gripper is front side of the platform this one is directed according to gripper imposed movement by operational task. This behaviour is more natural and thus more comprehensible by the person who controls the robot. It should be noted however that this assessment must be considered subject to a finer study of the influence of weights choice of the two secondary tasks in competition.

\subsubsection{Zone: close obstacle}

The mobile manipulator is close to the seizure zone but at a distance lower than threshold 2. In the example, threshold 2 is fixed at $5 \mathrm{~cm}$ from an obstacle. The obstacle can be the table on which the object is posed as it is shown in Figure 10. The initial situation of platform is given by its position $\left[x_{P}, y_{P}\right]^{T}=[1.35,1]^{T}(m)$ and its orientation $\theta_{P}=0^{\circ}$ (Figure 10). Arm extension is $40 \mathrm{~cm}$ in platform front side direction, $q_{a i}=[270,135,8,240]^{T}$ in degrees, $i=1$ to 3 . Only the mobile platform and the first three generalized coordinates of the arm are managed by autonomous functions for exploiting redundancy. As seen before the orientation and the position of the gripper is teleoperated by the user. 
The gripper is located initially on robot front side. The imposed operational task consists in reaching a goal placed on the table. Several positions of the objective were checked. Redundancy is exploited for getting an optimal manipulability from optimized measure point of view. There is no obstacle avoidance but platform linear velocity is imposed a null value. Two arm manipulability measures are evaluated here $\left(w, w_{d}\right)$.

Evolution of $w$ is used as reference to characterize the arm manipulation capacities in a given configuration. For each case, the end-effector trajectory, the arm manipulability evolution and the platform orientation are given. The interest to give the orientation of the platform is to evaluate the aptitude of the method to draw maximum benefit from the capacities of extension from the mobile manipulator.

Indeed as manipulator arm is fixed on the platform rear part, the reversal of this one permits to reach goals situated at longer distance from the system.

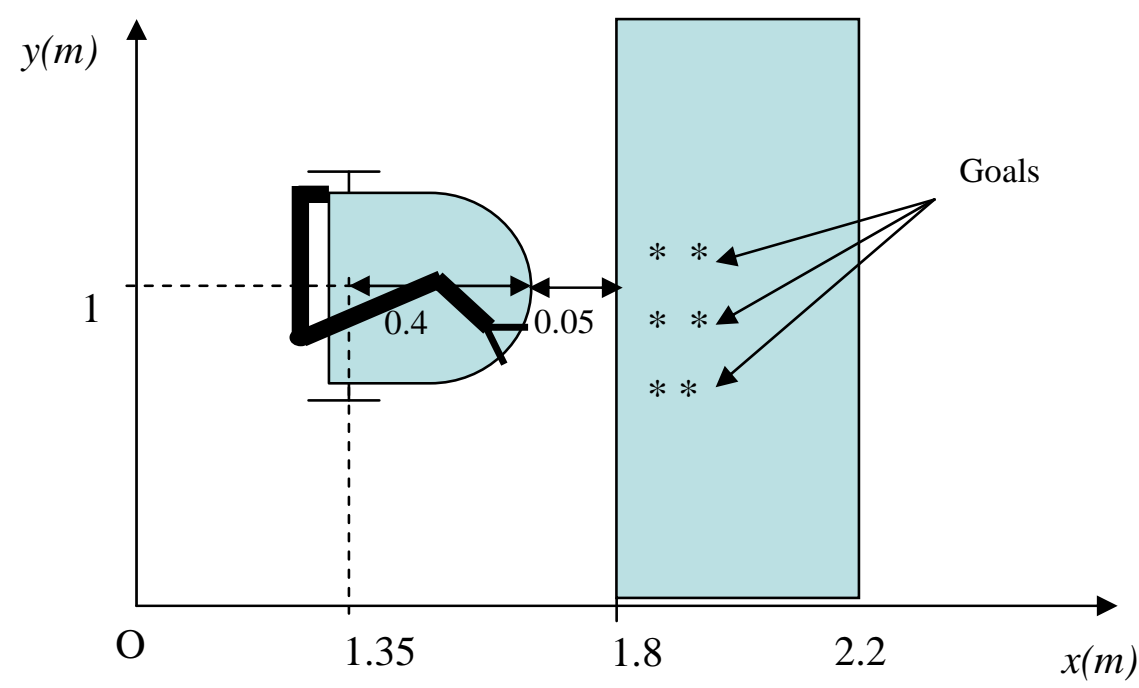

Figure 10 : Mobile platform at $5 \mathrm{~cm}$ from seizure table.

The goal is to reach an object situated at $20 \mathrm{~cm}$ of the border of the table. Gripper situation in 3D space is given by the vector $X_{b u t}=[2,1.2,0.4]^{\mathrm{T}}$. The objective is not reachable by manipulator arm alone without a platform move.

\section{a. Arm manipulability $w$ optimization.}

On Figure 11a and Figure 11b the results are given while optimizing $w$. End-effector trajectory given by figure 10a shows that EE reached its goal with a good manipulability (Figure 11b). Final extension of the manipulator arm is given by $X_{a f}=[0.52,0.32,0]^{\mathrm{T}}$ (arm not completely extended). The final orientation of mobile platform is $89.4^{\circ}$, which shows the rate intervening of the platform in task execution.

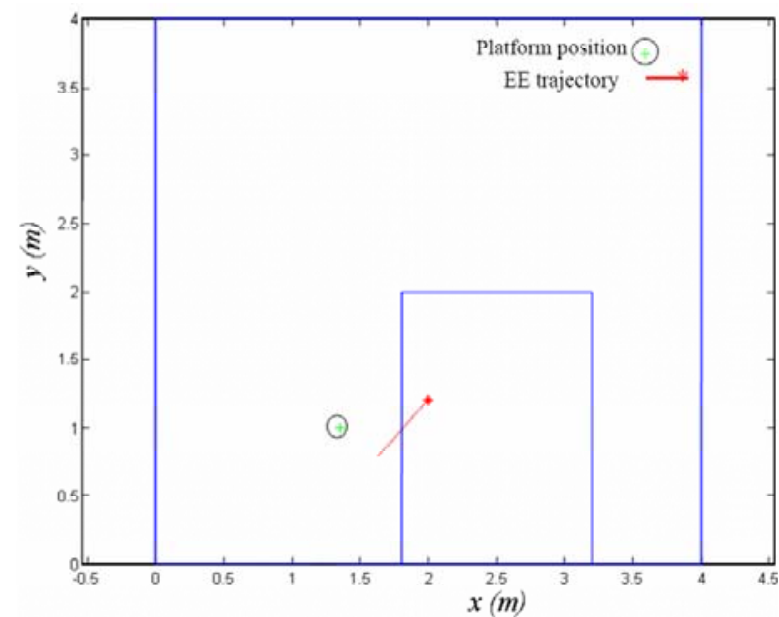

(a)
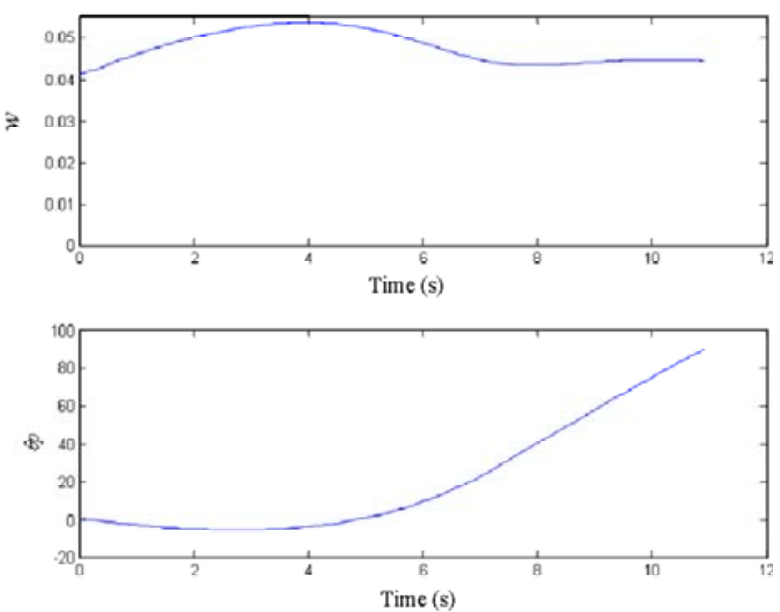

(b)

Figure 11 : Arm manipulability $w$ optimization a) EE trajectory, b) manipulability measure $w$ and platform orientation 


\section{b. Arm directional manipulability $w_{d}$ optimization}

Figure 12a and Figure 12b show the results while optimizing arm directional manipulability $w_{d}$. It is noted that the manipulability of the arm worsens gradually.

The final operational coordinates of the arm are given by $X_{a f}=[0.46 \mathrm{~s}, 0.46,0]^{\mathrm{T}}$ and the final platform orientation is $76^{\circ}$.

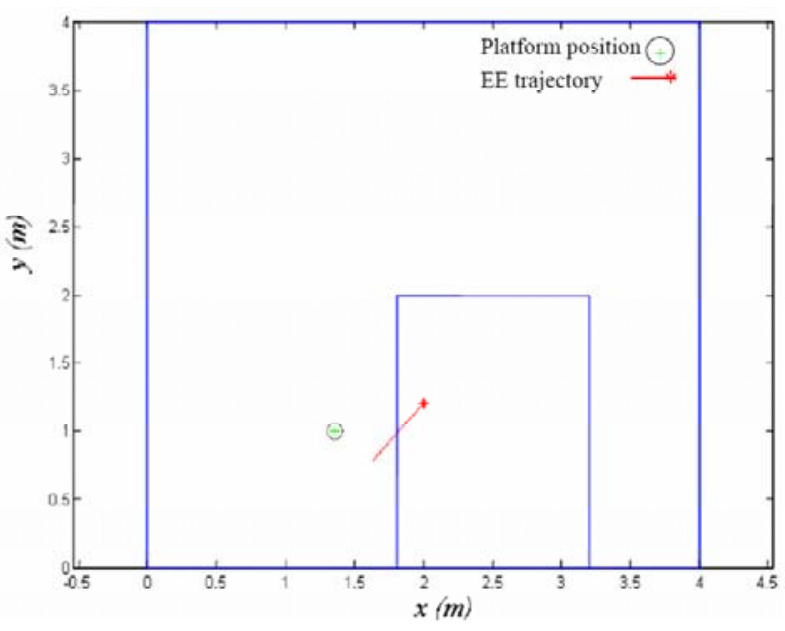

(a)
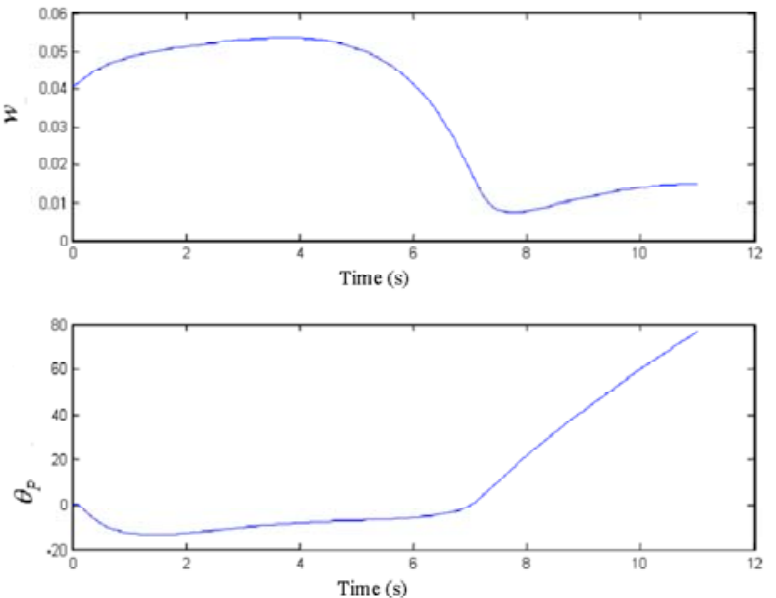

(b)

Figure 12: Arm directional manipulability wd optimization: a) EE trajectory, b) Evolutions of manipulability measure $w$ and platform orientation.

\section{Discussion}

We have evaluated the algorithm in different conditions. Examples that have been chosen to illustrate the mobile manipulator behaviour are representative. The different manipulability measures are compared according to the three criterions previously presented, task success, arm manipulation capacity at the end of task and platform behaviour. With regard to the platform, only the orientation is concerned since the linear speed is forced to zero. Desired task is achieved with success in any case in which the object is accessible. In the case where the object is not accessible, even with some appropriate movement of the mobile platform, at the end of the task the effector is as near as possible to the objective.

With regard to the manipulation ability of the arm there is no significant difference between results obtained in the case of an object situated in arm reachable space. When the objective is not reachable by the manipulator arm alone, the optimization of w preserves a good manipulability of the arm manipulator.

The linear displacement of the platform being forbidden, it is while playing on its orientation that the platform can increase reachable domain of the arm to reach an object out of range. In the example the object is not reachable by only the movement of the arm manipulator. The orientation of the platform permits then to seize the object. Results of simulation make appear that in phase "close objective" and in zone "close obstacle" w measure makes it possible to get a satisfactory answer to the three criteria while being superior to the other measures with regard to the second criteria.

In summary, in phase objective close, $w_{d}$ offers an appropriate answer to criteria for the zone "far obstacle" whereas $w$ answers to criteria for the zone "close obstacle". It is necessary to consider a change of the optimizing measure at the time the robot goes through one zone to another.

\subsubsection{Zone changing in phase close objective}

An example illustrates the change of zone that corresponds to the crossing of the threshold 2 . The protocol is identical to the one followed previously. By taking into account the previous results, one optimizes $w_{d}$ in zone far from an obstacle and $w$ in zone close from an obstacle. The initial situation of the platform is given by its position $\left[x_{p}, y_{p}\right]^{T}=[0.4,1](m)$ and its orientation $\theta_{P}=0^{\circ}$. The initial configuration of the first three generalized coordinates of the arm is $q_{a i}=[270,135.8,240]^{T}$ in degrees, $\mathrm{i}=1$ to 3 . The goal is situated at $X_{\text {Goal }}=[2,1.5,0.4]^{\mathrm{T}}$ at $20 \mathrm{~cm}$ from the border of the table. The end effector must follow a straight line with a constant speed $0.05 \mathrm{~m} / \mathrm{s}$. Figure 13a shows platform and end-effector trajectories. Imposed operational task is achieved correctly. Final objective is reached. Arm manipulability remains good (Figure 13b) during all the phase. In far zone, platform moves progressively to avoid the obstacle (Figure 13b). When it arrives near obstacle (close obstacle zone begins at $\mathrm{t}=28 \mathrm{~s}$ ), the linear displacement of the platform is forbidden. One notices on the curves of Figure 13b that changes of evolution of $w$ and the mobile platform orientation correspond to a change of the system behaviour. 
Platform contributes with its orientation ability to reach the objective with a good manipulability configuration. Final orientation of the platform is $\theta_{P f}=41^{\circ}$.

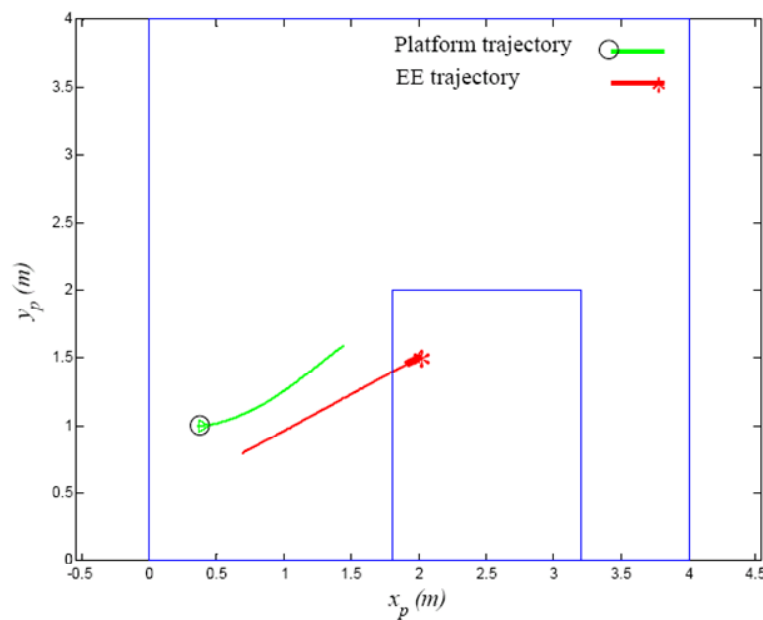

(a)
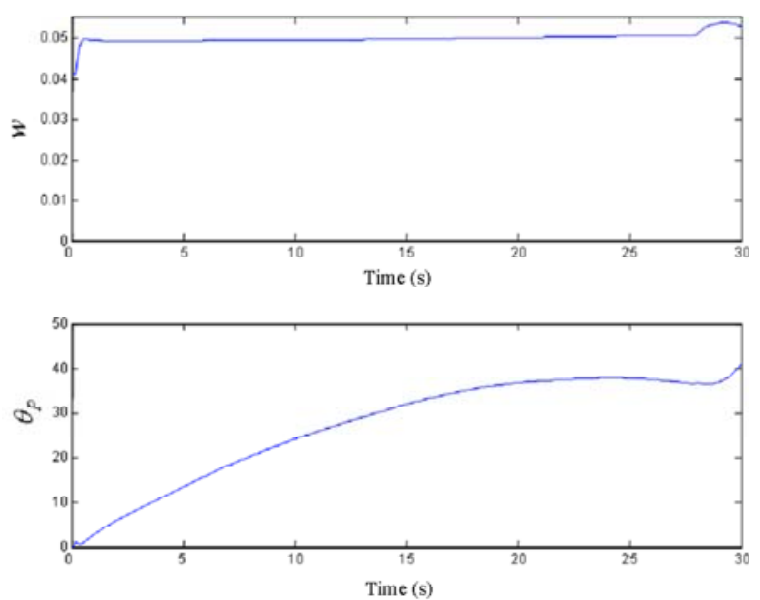

(b)

Figure 13 : manipulation and object seizure phase: a) platform and EE trajectories, b) manipulability measure $w$.

\subsection{Global example}

\subsubsection{Experimental protocol}

To evaluate all the phases, we consider that the mobile manipulator evolves in a room of $64 \mathrm{~m}^{2}$. The environment includes three obstacles and a table on which is put down the object to reach. The operational task imposed to the gripper consists of achieving the final goal while passing the proximity of the three sub-goals whose positions are known.

Sub-goals are situated in 3D space respectively at $X_{S G 1}=[2 ; 2.4]^{T}, X_{S G 2}=[4.5 ; 2.3]^{\mathrm{T}}$ and $X_{S G 3}=[7 ; 6 ; 0.4]^{\mathrm{T}}$. Final goal is located at $X_{\text {Goal }}=[7.1 ; 7.2 ; 0.4]^{\mathrm{T}}$. The passage from Phase "far objective" to phase "close objective" is activated as soon as the distance between gripper and final objective becomes lower than $50 \mathrm{~cm}$.

\subsubsection{Simulation}

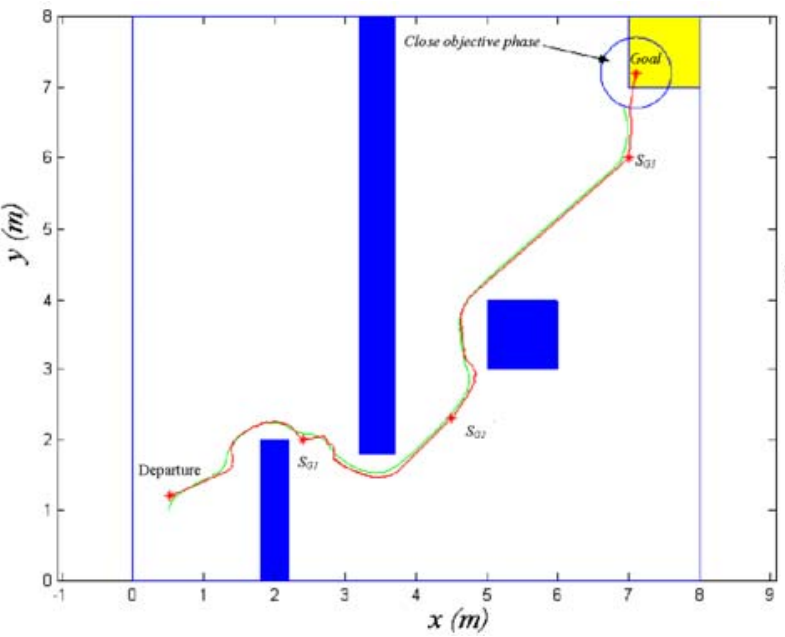

(a)

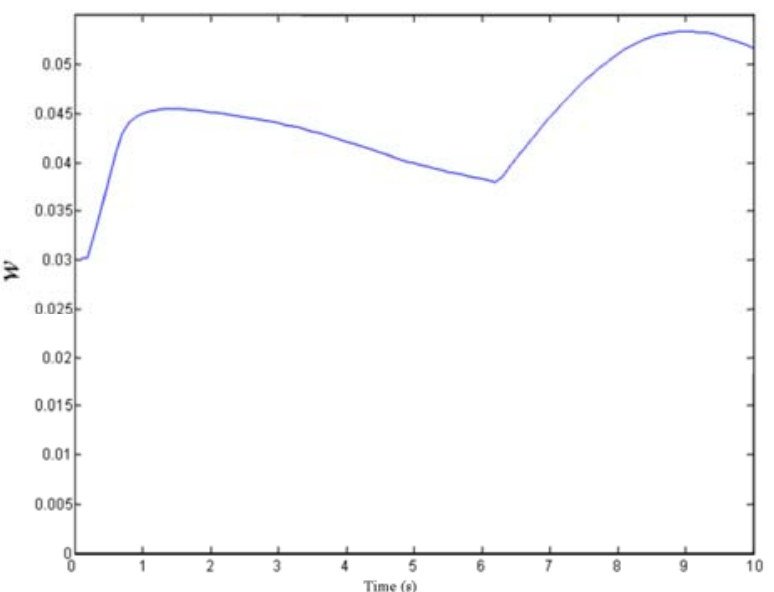

(b)

Figure 14 : Global example: a) EE and platform trajectories, b) manipulability measure $w$.

Figure 14a represents the trajectory of the end effector (which joins the sub-goals represented by stars) and of the platform for a complete mission in a complex environment. Initially the system is in phase "far objective" and in zone "far obstacle". To accomplish the task it starts with heading toward $S_{G 1}$ while following a straight line. When robot crosses threshold T1, reactive behaviour for obstacle avoidance is activated. These two behaviours of the robot go on up to phase "close objective". In this last phase, manipulation capacities of the manipulator arm are implied to accomplish the task correctly. 
On Figure 14b is given the manipulator arm manipulability w in the phase "close objective". The change of zones appears also on the manipulability curve. The objective is not reachable in good configuration by the arm alone. Association of platform orientation ability and the arm degrees of freedom, in close obstacle zone, permits to reach the objective with good configurations form manipulation point of view.

\subsubsection{Discussion}

We have evaluated the whole strategy for various positions of final objective, various values of the threshold 1 between phase "far objective" and phase "close objective" (variable threshold between $50 \mathrm{~cm}$ and 1 meter). The example which was selected to illustrate the mobile manipulator behaviour is representative. The system evolves from its starting point to its arrival point while passing by the different zones described in this paper. We used two manipulability measures. In certain situations, the imposed task and the constraints optimization induce the reverse gear movements of the platform. These situations are avoided by optimizing the directional manipulability of the arm. If the objective is close, the movements of the arm and the platform are coordinated to have a natural behaviour like human one. However if the objective is far, it is more interesting to use just locomotion capacity of the system. Arm is used only when it is necessary.

\section{Experimental results}

Autonomous functions have been separately evaluated on the physical robot i.e. obstacle avoidance (Hoppenot, 1996) and redundancy exploitation (Nait-Chabane, 2007). We only present two examples: i) a comparison of the results between the simulated and the real robot in case of the optimisation of the manipilability $w$, ii) the optimisation of the manipilability $w_{d}$ with the real robot.

The protocol remains the same as in the simulation (section 6). The initial situation of platform is given by its position $\left[x_{p}, y_{p}\right]^{T}=[0,0](m)$ and its orientation $\theta P=0^{\circ}$. The initial configuration of the first three generalized coordinates of the arm is $q_{a i}=[270,135.8,240]^{T}$ in degrees, $\mathrm{i}=1$ to 3 . The goal is situated at $X_{\text {Goal }}=[2,1.5,0.4]^{\mathrm{T}}$ at $20 \mathrm{~cm}$ from table border.

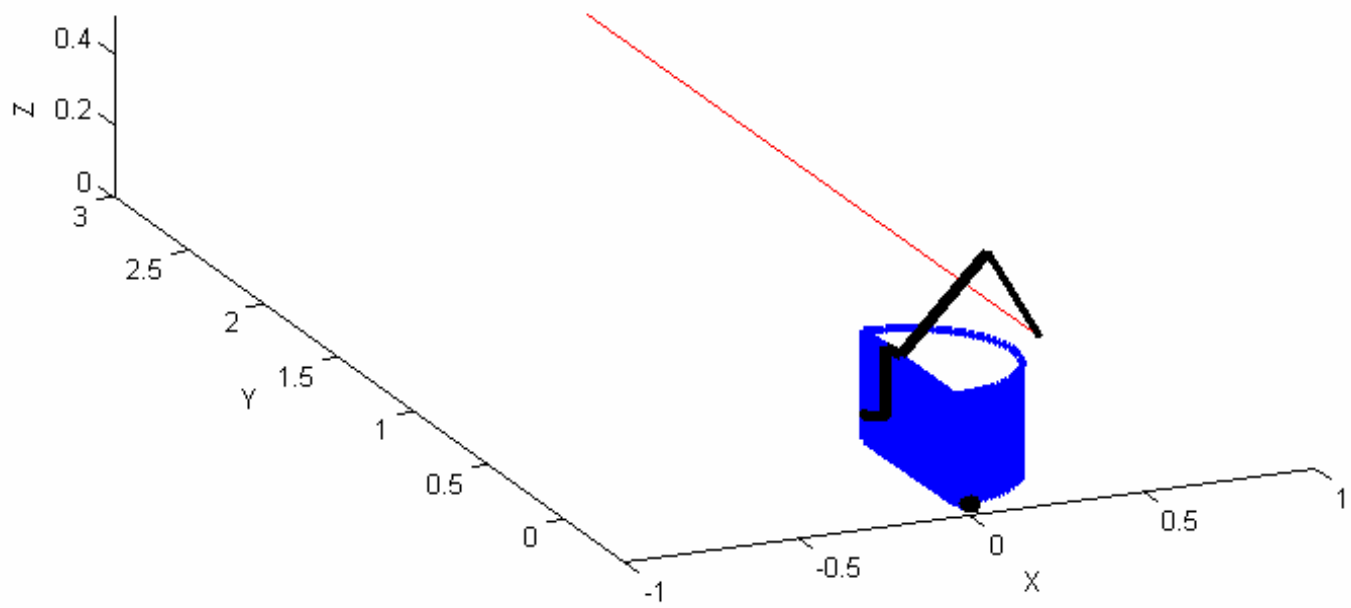

Figure 14: Experimental conditions

Operational task imposes that the end effector follows a straight line along y axis of the world frame with a constant speed $0.05 \mathrm{~m} / \mathrm{s}$ (fig. 14). The comparison aims at verifying that simulation and real robot results are very close. We have tested the algorithm for various measures of manipulabilty. The example to illustrate the comparison mobile manipulator is representative of all the evaluations we have carried out. In the example $w$ is optimized.

\subsection{Simulation results}

Figure 15a shows platform and EE trajectories. The platform moves back a little at the beginning to contribute to the improvement of the arm manipulability. Once the arm manipulability is improved, the platform goes forward. The arm manipulability (Figure 15b) is degraded slightly so the platform must reverse for continuing task while maintaining a good manipulability. EE carried out correctly the imposed task, but the platform behaviour due to non-holonomic constraint induces a reverse gear of the platform. 


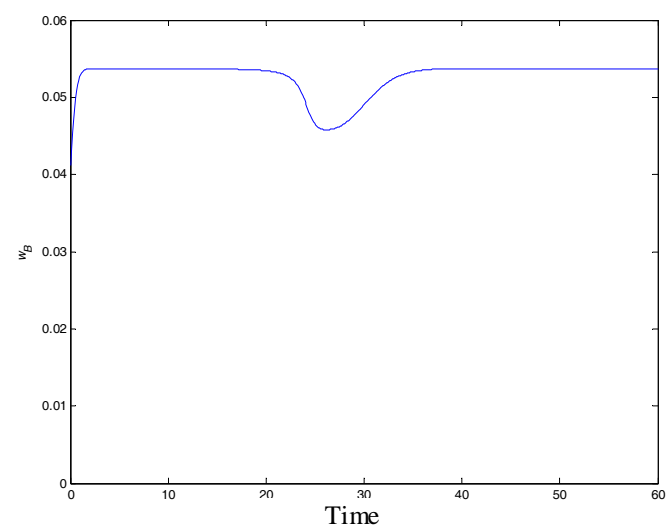

(a)

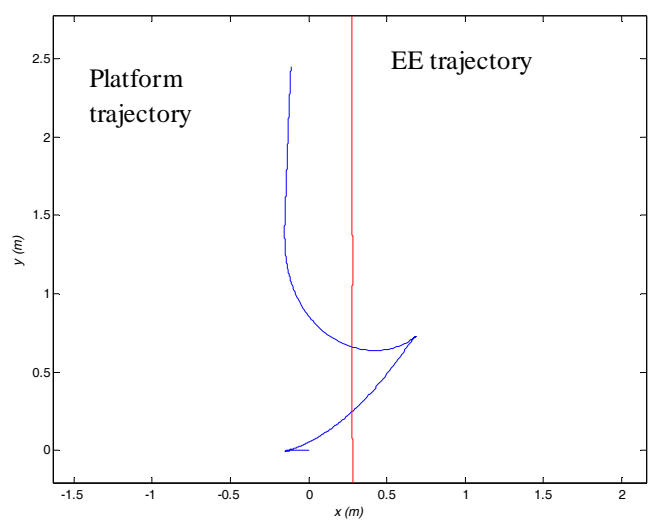

(b)

Figure 15: Simulation of the optimization of arm manipulability $w$ a) Manipulability measure $w$, b) Platform and EE trajectories.

\subsection{Real robot results}

\subsubsection{Comparison with the previous simulation}

We have implemented the same algorithm on the real robot. This operation is not easy. The heterogeneity of the communication protocols of the various components of the robot: Embarked PC, MANUS arm and mobile platform, have induced different response times to commands however sent simultaneously. It has been necessary to insert temporizations. The control of the platform is carried out in open loop. For the manipulator arm, a term of error is taken into account at each iteration to compensate for the error made during the execution of the preceding iteration.

Starting from a configuration given $q_{i}$, collected by the joint sensors, the operational task imposes that the end effector follows a straight line along y axis of the world frame with a constant speed $0.005 \mathrm{~m} / \mathrm{s}$ per $60 \mathrm{~ms}$ cycle.

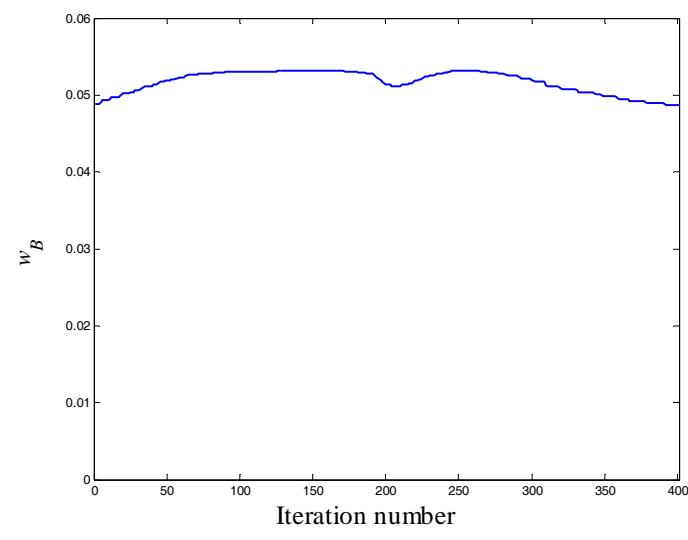

(a)

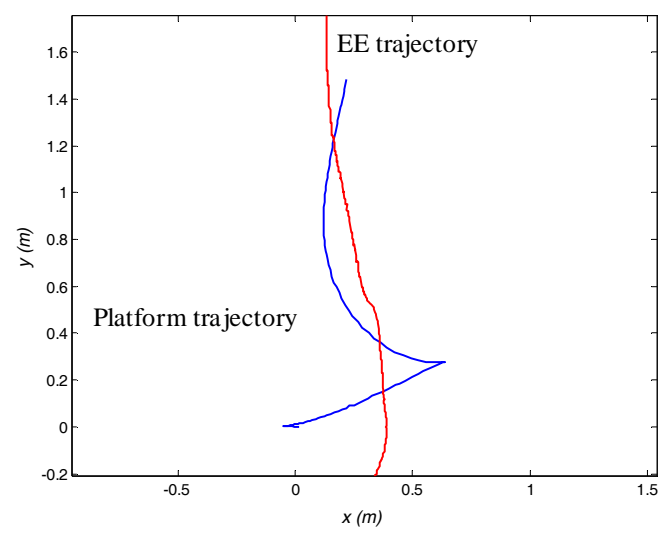

(b)

Figure 16: Optimization of arm manipulability $w$ with the physical robot a) Platform and EE trajectories, b)Manipulability measure $w$.

Figure 16a shows platform and EE trajectories. The shape of the curves is close to those given by simulation (Figure 15). The EE follows almost a straight line with an error which reaches $19 \mathrm{~cm}$ in end of the task. The error is mainly due to the experimental protocol. Indeed we only use dead reckoning for locating the platform.

\subsubsection{Improvement of real robot results}

We have improved experimental protocol and evaluated the other manipulability measure $w_{\mathrm{d}}$. The operational task imposes that the end effector follows a straight line with a constant speed $0.005 \mathrm{~m} / \mathrm{s}$ per $60 \mathrm{~ms}$ cycle. 


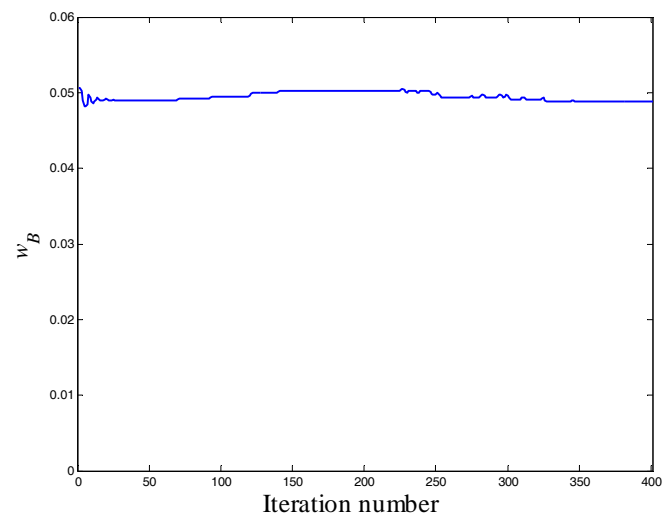

(a)

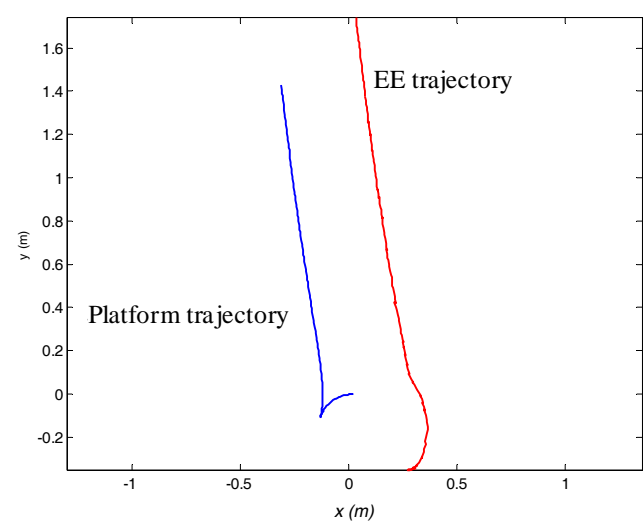

(b)

Figure 17: Optimization of arm manipulability $w_{d}$ with the physical robot a) Platform and EE trajectories, b)Manipulability measure $w$.

Figure 17a shows platform and EE trajectories. The EE follows the imposed straight line with an error less than $3 \mathrm{~cm}$ at end of the task. Figure 17b shows that the robot carries out the task imposed with a good manipulability of the manipulator arm. The error could still be reduced but it is important to remark that in the case of teleoperation this value is completely acceptable, which is not possible in autonomous robotics.

\section{Conclusion}

This paper proposed a strategy of displacement of a mobile manipulator whose principal objective is to place the system in the best configuration in terms of manipulability to carry out the final task of seizure. This strategy distinguishes the situations in which the task is carried out only by the platform, from the situations which require coordination between the platform and the manipulator arm. The idea is that the person worries only about the piloting of the end effector of the manipulator arm and that the remainder of the articulated system follows while taking into account if necessary the presence of obstacle. To facilitate man-machine co-operation we took as a starting point the human behaviour to establish a strategy divided into two phases according to whether the objective, namely the object to be seized, is far or close. In each phase, two zones are considered, far or close from an obstacle. Indeed the presence of obstacle must modify the behaviour of the system. The phase change is specified by the operator while the change of zone is automatically given thanks to the sensors of obstacle detection. For each phase, resolution method was defined making it possible to calculate the mobile manipulator controls using orders provided by the active control mode. In the first phase called far objective, the system is not redundant, the arm manipulator being inactive. Two behaviours are taken into account, convergence towards the objective (operational task) and obstacle avoidance. This last one is activated only near obstacles and becomes increasingly dominating as the system approaches the obstacle. In the second phase called close objective, the system becomes redundant enabling manipulator arm. It is then interesting to exploit this redundancy. The method suggested is based on the traditional use of pseudo-inverse with a term of optimization. For each case, we defined the primary task which is in the majority of the cases the operational task and when there was redundancy, the criterion to be optimized and/or the task secondary and if necessary the additional task. Simulations made it possible to check the overall strategy and also to compare several measures of manipulability when the possibility of exploiting the redundancy was possible. The comparison was done on the following points, success of the task, capacity of handling of the arm at the end of the task, behaviour of the platform. Measures relating to the only arm versus those on the mobile manipulator bring a better answer. $w$ for the phase "close objective" and the zone "close obstacle". $w_{d}$, the new measure which we proposed above for the phase "close objective" and the zone "far obstacle". Several constraints are possible to exploit redundancy. We have chosen to optimize the manipulability measure and the avoidance of obstacles, which answer to our aims. In the human robot cooperation that we propose, the autonomous functions of the robot can be regarded as a specific assistance of the user according to the situation defined by the phase of approach in which the robot is. This paper does not propose an autonomous path planning of the mobile arm but an assistance to the user for remote controlling it. The interest of this approach is to leave to the user the control of the robot while facilitating the task to him. 
K. Nait-Chabane, S. Delarue, P. Hoppenot and E. Colle: "Strategy of Approach for Seizure of An Assistive Mobile Manipulator" - Robotics and Autonomous Systems, vol. 57, pp 222,-235, 2009.

\section{References}

(Bayle, 2001a)

(Bayle, 2001b)

(Benreguieg, 1997)

(Bowling, 2005)

(Busnel, 2001)

(Chiaverini, 1997)

(Evers, 2001)

(Foulon, 1998)

(Foulon, 1999)

(Grasso, 1998)

(Hoppenot, 1996)

(Hoppenot, 2002)

(Jeannerod, 1984)

(Khatib, 1986)

(Kwee, 1993)

(Land, 1994)

(Mestre, 2005)

(Nait-Chabane, 2006)

(Nait-Chabane, 2007)

(Padois, 2007)

(Rybarczyck, 2002)
B. Bayle, J. Y. Fourquet, M. RENAUD. Manipulability analysis for mobile manipulators. International Conference on Robotics and Automation (ICRA'2001), Seoul (Korée), 2126 Mai 2001, pp.1251-1256.

B. Bayle, J. Y. Fourquet, M. Renaud. Using Manipulability with Nonholonomic Mobile Manipulators, 3rd International Conference on Field and Service Robotics (FSR'2001), Helsinki (Finlande), 11-13 Juin 2001, pp.343-348.

M. Benreguieg. Aides à la navigation d'un robot mobile dans un environnement partiellement connu. Thèse de Doctorat de l'Université d'Evry Val d'Essonne, 16 janvier 97.

Bowling, A, Khatib, O, "The dynamic capability equations: A new tool for analyzing manipulator dynamic performance.", IEEE Transactions on Robotics, in press. Volume 21, Issue 1, Feb. 2005 Page(s):115 - 123

M. Busnel, R. Gelin and B. Lesigne, "Evaluation of a robotized MASTER/RAID workstation at home: Protocol and first results”, Proc. ICORR 2001, Vol. 9, pp. 299-305, 2001.

S. Chiaverini. Singularity-Robust Task-Priority Redundancy Resolution for Real-Time Kinematic Control of Robot Manipulators. IEEE Transactions on Robotics and Automation, Vol. 13, No. 3, June 1997, pp. 398-410.

H. G. Evers, E. Beugels and G. Peters, "MANUS toward new decade”, Proc. ICORR 2001, Vol. 9, pp. 155-161, 2001.

G. Foulon. Génération de mouvements coordonnés pour un ensemble d’une plate-forme mobile à roues et d'un bras manipulateur. Thèse de Doctorat Institut National des Sciences Appliquées, Toulouse, soutenu le 05/10/1998.

G. Foulon, J. Y. Fourquet, M. RENAUD. Coordinating mobility and manipulation using nonholonomic mobile manipulators, Control Engineering Practice 7 (1999) 391-399.

R. Grasso, P. Prévost, Y.P Ivanenko \& A. Berthoz. Eye-head coordination for the steering of locomotion in humans: An anticipatory synergy. Neuroscience Letters, 253,115-118.

P. Hoppenot , M. Benreguieg, H. Maaref., E. Colle. and C. Barret: "Control of a medical aid mobile robot based on a fuzzy navigation" - IEEE Symposium on Robotics and Cybernetics, Lille, France, pp. 388-393, july 1996.

P. Hoppenot, E. Colle. Mobile robot command by man-machine co-operation Application to disabled and elderly people assistance. Journal of Intelligent and Robotic Systems, vol. 34, n³, pp. 235-252, July 2002.

M. Jeannerod. The timing of natural prehension movements. Journal of Motor Behavior, 16, 235-254.

O. Khatib. Real-Time Obstacle Avoidance For Manipulators and Mobile robots. International Journal of Robotic Research. Vol.5, no 1, pages 90-98 ; 1986.

H. Kwee, C.A. Stanger, “The Manus robot arm”, Rehabilitation Robotics News letter, Vol. 5, No 2, 1993.

M.F Land, N. Mennie \& J. Rusted. The roles of vision and eye movements in the control of activities of daily living. Perception, 28, 1311-1328.

D.R. Mestre, Y. Rybarczyk, P. Hoppenot, E. Colle, : "Assistance Robotics: Implementation of human-like visuo-motor synergies on a teleoperated mobile device". CSUN's 20th Annual International Conference "Technology and Persons with Disabilities" March 14-19, 2005, Los Angeles, CA.

K. Nait-Chabane, P. Hoppenot, E. Colle. Mobile arm for disabled people assistance Manipulability measure for redundancy solve. - Journal of Intelligent \& Robotics Systems, received 28 July2005, accepted 3may2006.

K. Nait-Chabane, P. Hoppenot and E. Colle, "Directional Manipulability for Motion Coordination of An Assistive Mobile Arm”, Fourth International Conference Informatics in Control, Automation and Robotics, Vol. 2, pp 201-208, Angers, France, May 9-12, 2007.

V. Padoisa, J.-Y. Fourqueta and P. Chirona; "Kinematic and dynamic model-based control of wheeled mobile manipulators: a unified framework for reactive approaches Robotica (2007), 25:157-173 Cambridge University Press Copyright Cambridge University Press 2007.

Y. Rybarczyk, E. Colle, P. Hoppenot: "Contribution of neuroscience to the teleoperation of rehabilitation robot", SMC'2002, Hammanet, Tunisia, 6-9 october 
K. Nait-Chabane, S. Delarue, P. Hoppenot and E. Colle: "Strategy of Approach for Seizure of An Assistive Mobile Manipulator" - Robotics and Autonomous Systems, vol. 57, pp 222,-235, 2009.

(Salisbury, 1982) J. K. Salisbury and J.J. Craig, "Articulated hands: Force Control and Kinematic Issues,” Intl. J. Robotics Research, Vol. 1, no. 1, pp. 4-17, 1982.

(Van der Loos, H.F.M. Van der Loos, "VA/Stanford Rehabilitation Robotics Research and Development 1995) Program: Lessons Learned in the Application of Robotics Technology to the Field of Rehabilitation,” IEEE Trans. Pn Rehabilitation Engineering, Vol. 3, nº1, pp. 46-55, March 1995.

(Yoshikawa, 1985) T. Yoshikawa, "Manipulability of Robotic Mechanisms," International Journal of Robotics Research, 1985, Vol. 4, no. 2, pp. 3-9.

(Yoshikawa, 1990) T. Yoshikawa. Foundation of robotics: Analysis and control. The MIT Press, 1990. 